\title{
The treatment of Sleep Apnea Syndrome: looking for new answers for old problems
}

\author{
F. Fanfulla1, A. Braghiroli2
}

Monaldi Arch Chest Dis 2004; 61: 3, 138-139.

Keywords: Autotitrating Positive Airway Pressure; Continuous Positive Airway Pressure; Obstructive Sleep Apnea; Polysomnography.

“Salvatore Maugeri” Foundation, IRCCS, Sleep Laboratory, Scientific Institutes of Montescano ${ }^{1}$ and Veruno ${ }^{2}$, Italy.

Correspondence: Francesco Fanfulla, MD; Laboratorio di Polisonnografia; Istituto Scientifico di Montescano IRCCS; Fondazione Salvatore Maugeri; 27040 Montescano (Pavia) - Italy; e-mail; ffanfulla@fsm.it

Obstructive sleep apnea syndrome (OSA) is characterized by repeated episodes of upper airway occlusion during sleep, resulting in transient asphyxia until an arousal restores airway patency. The consequences of these events are profound, including sleep fragmentation, increased blood pressure, daytime sleepiness, quality of life impairment and a greater risk of motor vehicle accidents.

Continuous positive airway pressure (CPAP) therapy is generally considered as the first-line treatment for OSA. Standard CPAP devices deliver a constant positive pressure that acts as a pneumatic splint, preventing upper airway collapse. In patients with OSA, CPAP is highly effective in eliminating obstructive events, decreasing blood pressure, reducing sleepiness and improving quality of life [1-2]. CPAP levels are generally set during an attended laboratory polysomnography to eliminate apneas, hypopneas, snoring, and flow limitation in all positions and sleep stages [3]. Therefore, since pressure requirement will change during the night, according to sleep stage and body position, the set pressure is usually higher than the level actually able to maintain airway patency for at least a portion of the night [4]. The use for the whole night of a pressure level higher than the minimum needed could potentially increase mask leaks, mouth leaks, pressure intolerance, and theoretically reduce acceptance and adherence to treatment in some patients.

Autoadjusting CPAP devices (APAP) have been developed with the aim of solving these problems. APAP systems deliver a variable pressure during the night, theoretically according to the actual need of patients. Every APAP device relies on the recording of a physiological variable to detect upper airway obstruction and changes the pressure delivered according to specific algorithms. APAP devices are generally designed to progressively increase pressure till obstructive events disappear and then to decrease pressure if no events are detected over a set period of time, so that the mean pressure applied across the night is generally lower than that applied with fixed CPAP. It has been hypothesized that this reduction in mean applied pressure may improve patient's tolerance and adherence to treatment in comparison with fixed CPAP therapy. Most of the studies available do not support this hypothesis and a recent meta-analysis [5] concludes that APAP and fixed CPAP show similar efficacy in eliminating respiratory events and in improving subjective sleepiness; adherence to treatment overlaps despite the lower mean pressure delivered using APAP. Considering the higher costs of APAP devices a systematic prescription in every OSAS patient lacks of scientific support. However, some of the studies have been carried out in a single night and some patients require several days before becoming fully accustomed to the device.

In the current issue of Monaldi Archives a paper by Resta and coworkers [6] focuses on the effectiveness of APAP versus traditional CPAP therapy after one month of use with a particular attention on the arousability. The twenty OSAS patients included in the study were randomized to receive APAP or fixed CPAP therapy at home; the polysomnography performed after one-month therapy, revealed no differences between the two treatment in sleep architecture, respiratory function and daytime sleepiness. Furthermore, the compliance was similar in the two groups of patients. The authors' conclusion is in line with the above mentioned meta-analysis [5]. They underline that there is no basis for a higher cost therapy, although the risk of an excessive arousal index with APAP, showed in a single night setting by some of the authors in a previous study [7], has not been confirmed.

The higher cost of APAP should not be considered the only disadvantage compared to CPAP. The principle of autotitration requires both a negative and a positive feed-back. Leaks through the mouth opened or if the mask is occasionally dis- 
placed can cause a malfunctioning of the positive feed-back and could possibly bring to the paradox of an occasionally higher-than-necessary pressure delivered by APAP in the home setting. Since the design of the study by Resta et al [6] required an in-lab recording at follow up, the occurrence of this event cannot be quantified since technician's supervision promptly corrects any mask displacement.

Other limitations of APAP should be bared in mind. The use of APAP is not indicated when OSA is associated with COPD, in OSA patients with a high number of central apneas (usually $>10$ events per hour), in patients with chronic heart failure, or in patients with previous uvulopalatopharyngoplasty (UPPP) when the APAP algorithm is based on snoring detection (8-9).

Another finding of the paper by Resta et al [6] deserves attention. The mean usage of the device - both continuous or autoadjusting - is limited to about 5 hours. This result is quite consistent with the literature coming from any part of the world and underlines a sort of reluctance of most patients to the use for the entire sleep period. APAP does not grant any improvement from this point of view. The relief of symptoms is obviously obtained with these figures or patients would spontaneously use the device more, but we are well aware that a resolution of daytime somnolence can occur with subtherapeutic CPAP without changing the risk profile of adverse cardiovascular events $[10,11]$.

What is the present role of APAP after all? Surely APAP may be useful in long term treatment of some sub-group of patients, i.e. patients with a very high variability of effective pressure during the night (postural apneas, REM related OSA, vasomotor rhinitis) or perhaps with a nightto-night variability (i.e.: shift working). However the real time and cost decreasing role of APAP seems to profile in CPAP titration. Its usage in the supervised sleep lab setting is already a standard [8], but even in unattended setting its role is under investigation. A recent co-operative Spanish study [12] shows that a training period at home is able to identify the therapeutic CPAP level very closely to the figure obtained with standard in-lab titration. This result cannot be transferred to any type of APAP, on the contrary some of the autoadjusting devices designed for therapeutic purpose seem to perform poorly when used to identify a fixed CPAP therapeutic pressure [13]. Resta et al [6] used the same device of the Spanish study and found that the 95th percentile CPAP delivered in the month of treatment overlapped the in-lab titrated CPAP pressure "despite variable individual differences". How many patients will not be properly treated if we rely only on the report of an automatic device? We need more stud- ies and a careful cost-benefit analysis before evidence can lead to a drastic change in titration procedures.

\section{References}

1. Malhotra A, Ayas N, Epstein L. The art and science of continuous positive airway pressure therapy in obstructive sleep apnea. Curr Opin Pulm Med 2000; 6: 490-5.

2. Patel SR, White DP, Malhotra A, Stanchina M, Ayas NT. The effect of CPAP therapy on subjective and objective sleepiness in obstructive sleep apnea: a metaanalysis of randomized controlled trials. Arch Intern Med 2003; 163: 565-71.

3. Montserrat JM, Ballester E, Olivi H. Time course of stepwise CPAP titration. Behaviour of respiratory and neurological variables. Am J Respir Crit Care Med 1995; 152: 1854-9.

4. Oksenberg A, Silverberg DS, Arons E, Radwan H. The sleep supine position has a major effect on optimal nasal continuous positive airway pressure: relationship with rapid eye movements and non-rapid eye movements sleep, body mass index, respiratory disturbance index, and age. Chest 1999; 116: 1000-6.

5. Ayas NT, Patel SR, Malhotra A, et al. Auto-titrating versus standard continous positive airway pressure for the treatment of Obstructive Sleep Apnea: results of a meta-analysis. Sleep 2004; 27: 249-53.

6. Resta O, Carratu' P, Depalo A, et al. Effects of fixed compared to automatic CPAP on sleep in Obstructive Sleep Apnea Syndrome. Monaldi Arch Chest Dis 2004; 61: 153-156.

7. Marrone $\mathrm{O}$, Insalaco $\mathrm{G}$, Bonsignore MR, Romano S, Salvaggio A, Bonsignore G. Sleep structure correlates of CPAP variations during application of an autotitration CPAP machine in obstructive sleep apnea syndrome. Chest 2002; 121: 759-767.

8. Littner M. Hirshkowitz M, Davila D, et al. Practice parameters for the use of auto-titrating continuous positive airway pressure devices for titrating pressures and treating adult patients with Obstructive Sleep Apnea Syndrome. Sleep 2002; 25: 143-147.

9. Berry RB, Parish JM, Hartse KM. The use of autotitrating continuous positive airway pressure for treatment of adult Obstructive Sleep Apnea. Sleep 2002; 25: 148-173.

10. Pepperell JC, Ramdassingh-Dow S, Crosthwaite N, et al. Ambulatory blood pressure after therapeutic and subtherapeutic nasal continuous positive airway pressure for obstructive sleep apnoea: a randomised parallel trial. Lancet 2002; 359: 204-10

11. Becker HF, Jerrentrup A, Ploch T, et al. Effect of nasal continuous positive airway pressure treatment on blood pressure in patients with obstructive sleep apnea. Circulation 2003; 107: 68-73.

12. Masa JF, Jimenez A, Duran J, et al. Alternative methods of titrating continuous positive airway pressure: a large multicenter study. Am J Respir Crit Care Med 2004; 170: 1218-24.

13. Kessler R, Weitzenblum E, Chaouat A, Iamandi C, Alliotte T. Evaluation of unattended automated titration to determine therapeutic continuous positive airway pressure in patients with obstructive sleep apnea. Chest 2003; 123: 704-10. 